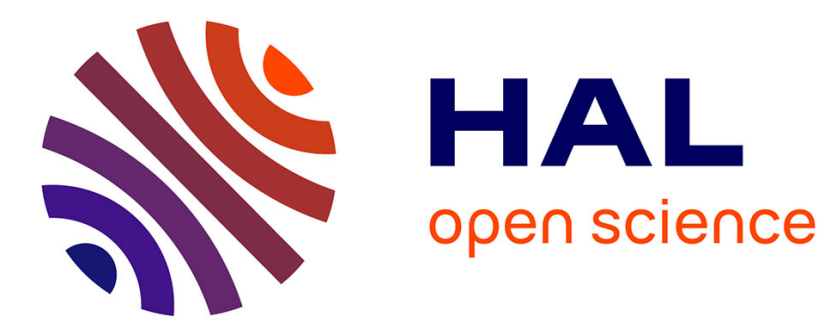

\title{
Tailoring of the porous structure of soft emulsion-templated polymer materials
}

Artem Kovalenko, Kévin Zimny, Benoit Mascaro, Thomas Brunet, Olivier

Mondain-Monval

\section{- To cite this version:}

Artem Kovalenko, Kévin Zimny, Benoit Mascaro, Thomas Brunet, Olivier Mondain-Monval. Tailoring of the porous structure of soft emulsion-templated polymer materials. Soft Matter, 2016, 12 (23), pp.5154-5163. 10.1039/c6sm00461j . hal-02890725

\section{HAL Id: hal-02890725 \\ https://hal.science/hal-02890725}

Submitted on 17 Jul 2020

HAL is a multi-disciplinary open access archive for the deposit and dissemination of scientific research documents, whether they are published or not. The documents may come from teaching and research institutions in France or abroad, or from public or private research centers.
L'archive ouverte pluridisciplinaire HAL, est destinée au dépôt et à la diffusion de documents scientifiques de niveau recherche, publiés ou non, émanant des établissements d'enseignement et de recherche français ou étrangers, des laboratoires publics ou privés. 


\section{Tailoring of the porous structure of emulsion-templated soft acoustic resonators}

Artem Kovalenko ${ }^{1 *}$, Kévin Zimny ${ }^{1}$, Benoit Mascaro ${ }^{2}$, Thomas Brunet ${ }^{2}$, Olivier Mondain-Monval ${ }^{1 *}$

${ }^{1}$ - University of Bordeaux - CNRS, Centre de Recherche Paul Pascal, Pessac, France

2 - University of Bordeaux - CNRS - INBP, Institut de Mécanique et d'Ingénierie, Talence, France

* - corresponding authors

\section{Sommaire}

Collapse of the porous structure of emulsion-templated soft acoustic materialsErreur ! Signet non défini.

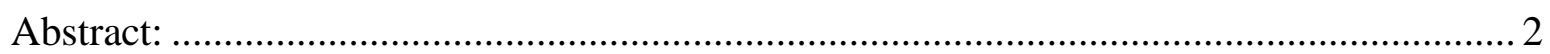

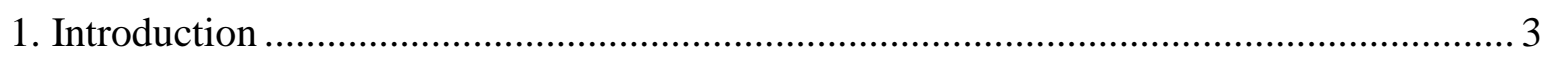

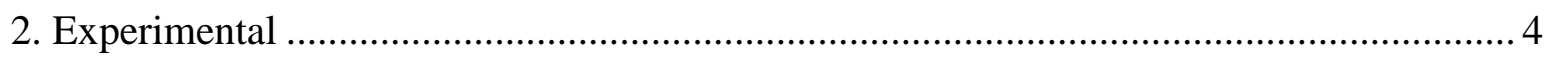

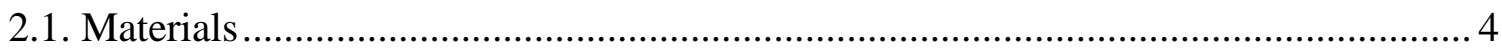

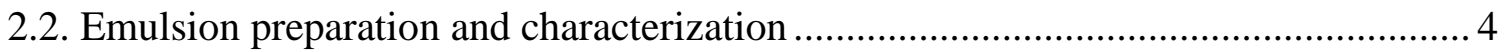

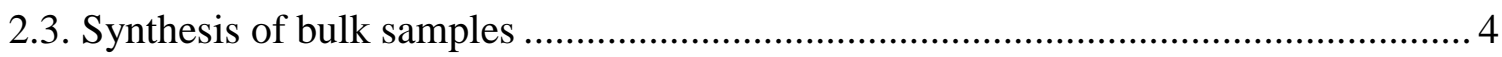

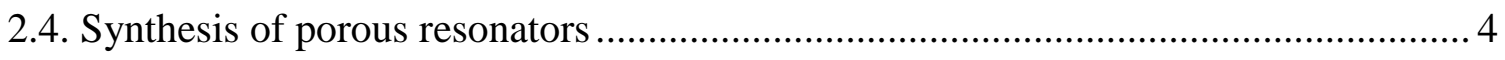

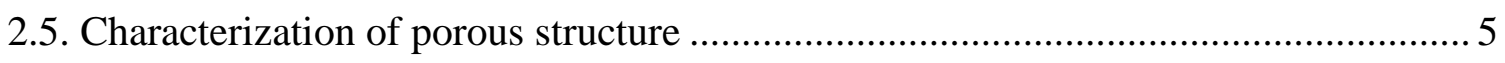

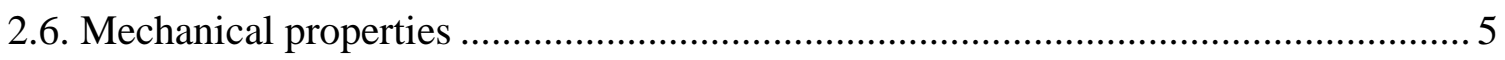

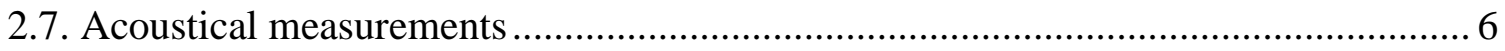

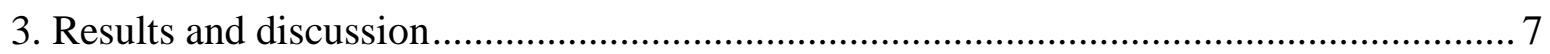

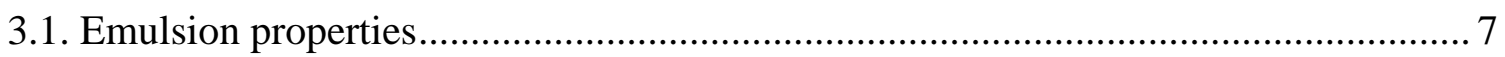

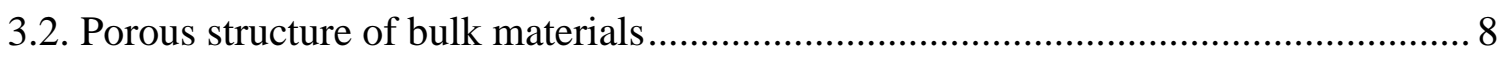

3.3. Mechanical and acoustical properties of bulk materials ........................................ 10

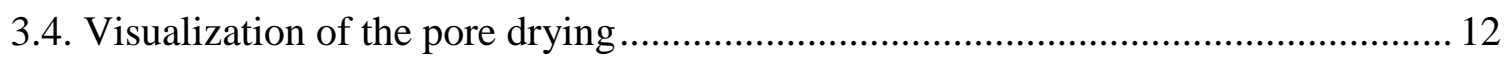

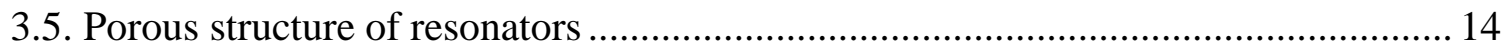

3.6. Mechanical and acoustical properties of resonators ................................................ 16

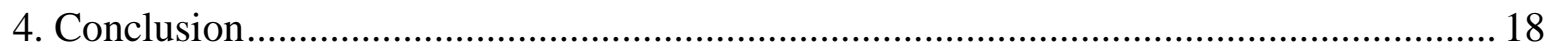

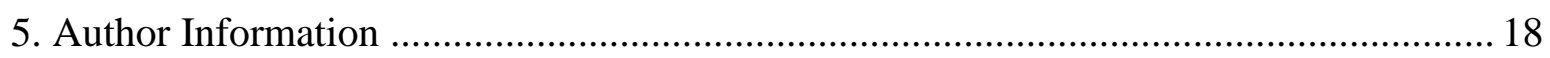

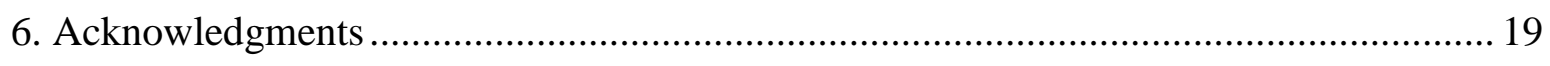

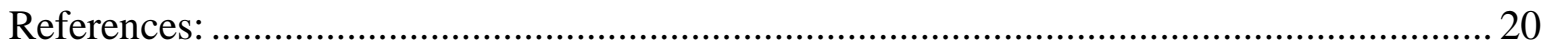




\begin{abstract}
:
This paper discusses the formation of soft porous materials obtained by the polymerization of inverse water-in-silicone (polydimethylsiloxane PDMS) emulsions. We show that the initial state of the emulsion has a strong impact on the porous structure and properties of the final material. The obtained soft and compressible porous polymers have very low internal sound speed and were recently used to build the micro-resonators entering the composition of a 3D acoustic metamaterial. We show that using surfactant with different solubility in the emulsion continuous phase (PDMS), it is possible to tune the interaction between emulsion droplets, which leads to materials with either interconnected or isolated pores. These two systems present completely different behavior upon drying, which results in macroporous air-filled materials in the interconnected case and in a collapsed material with low porosity in the second case. Finally, we compare the mechanical and acoustical properties of these two types of bulk polymer monoliths. We also describe the formation of beads in these two cases. We show that materials with interconnected macroporous structure have low mechanical moduli and low sound speed, and are suitable for acoustic applications. Mechanical and acoustical properties of the materials with collapsed porous structure are similar to that of the nonporous silicone, which makes them acoustically inactive.
\end{abstract}




\section{Introduction}

Gas-filled porous materials have unique thermal, mechanical, acoustical and transport properties that may be tuned by varying either the matrix composition or the porous structure. ${ }^{1}$ Porous materials with mineral, plastic, metal-organic, carbon matrices are widely used in various applications such as thermal isolation, hydrogen storage, shock wave absorption, catalysis, membrane filtrations, medical implants and tissue engineering $2,3,4,5,6$. Due to the presence of large proportion of gas, porous materials also present a high compressibility, which makes them interesting for acoustic applications. ${ }^{7}$ Among other properties, they exhibit very low sound speed, as for instance silica aerogels ${ }^{8}$ in which it may fall down to values as low as $100 \mathrm{~m} / \mathrm{s}$ (for porosities > 95\%). Similarly, it was also shown that porous silicone rubbers exhibit very low sound speed as soon as their porosity attains a few percent". Such "ultraslow" materials are key elements of locally resonant acoustic metamaterial. Indeed, as first shown by Li and Chan, 10 "ultraslow" inclusions can act as Mie resonators, when shaped as spheres and dispersed in a continuous matrix exhibiting a much higher sound speed. ${ }^{11}$ Both silica aerogels and porous silicone rubbers can be processed as micrometric beads using microfluidic techniques ${ }^{12},{ }^{13}$.

While several industrial procedures for obtaining elastomeric foams with millimeter-scale cells are known ${ }^{14}$, production of macroporous elastomers with micron-sized pores remains challenging. The synthesis strategy that was used for macroporous silicone , $^{15,16}$ is based on the polymerized High Internal Phase Emulsion method (polyHIPE) ${ }^{17,18}$. In this method, initially developed for stiff polymers (polystyrene-divinylbenzene (PS/DVB) ${ }^{19,20}$ ), the continuous phase of a water-in-oil emulsion is polymerized in order to obtain a wet solid with interconnected porous structure. In the case of PS-DVB, washing and drying of the solid leads to porous polymer materials in which the air volume fraction can be varied from $50 \%$ (sometimes referred as polyLIPEs or polyMIPEs ${ }^{21}$, corresponding to low or medium emulsion concentration) up to $90 \%$. The interconnections between the pores in such samples may occur due to mechanical stresses during the polymerization ${ }^{22}$ or the post-synthesis processing ${ }^{23}$.

In this paper we show that the initial emulsion structure is a key parameter controlling both mechanical and acoustic properties of polyMIPE silicone materials. Choosing an appropriate surfactant for inverse water/PDMS emulsions, we are able to obtain two different emulsion structures, with repulsive or attractive interactions between water droplets. By cross-linking the emulsions, we are able to produce silicone polyMIPE materials with isolated or interconnected porous structure. Bulk samples as well as spherical beads (resonators) are synthesized. We establish the link between the obtained porous structure and the mechanical and acoustical properties of the obtained materials. We reveal the importance of the interconnections between pores in maintaining high porosity of the soft silicone rubber during the drying process, which is crucial for the elaboration of acoustic Mie resonators. 


\section{Experimental}

\subsection{Materials}

UV-cross-linkable PDMS mixture was supplied by BlueStar Silicones. It is composed of epoxy-modified PDMS (Silcolease UV Poly200 ${ }^{\mathrm{TM}}$ ) and diaryl iodonium catalyzer (Silcolease UV Cata211 ${ }^{\mathrm{TM}}$ ). UV sensitizer (2-isopropylthioxanthone) was obtained from Rahn AG. We used two surfactants: lauryl PEG-8 dimethicone surfactant Silube ${ }^{\circledR}$ J208-812 (Siltech LLC) and 2-octyl-1-dodecanol (Sigma Aldrich). We used deionized MiliQ water and $\mathrm{NaCl}$ from Sigma Aldrich.

\subsection{Emulsion preparation and characterization}

The continuous phase of the emulsions was obtained by mixing $12 \mathrm{~g}$ of silicone based UV cross-linkable PDMS fluid together with $0.5 \mathrm{~g}$ of catalyzer, $10 \mathrm{mg}$ of UV sensitizer and $50 \mathrm{mg}$ of surfactant (Silube ${ }^{\circledR}$ J208-812, later referred to as Silube or 2-octyl-1-dodecanol, later referred to as $\mathrm{OH} 457$ ). In order to obtain $30 \%$ vol. emulsions, $5.4 \mathrm{~g}$ of water phase containing 1.5 wt. $\% \mathrm{NaCl}$ was introduced under mechanical stirring with a home-made helical device. The size distributions of emulsions were characterized both by static light scattering, optical microscopy and rheometry. Static light scattering experiments were performed in a Mastersizer S apparatus (Malvern) with dodecane as the continuous phase. In dodecane, emulsion droplets remain fully dispersed whatever the used surfactant. For optical measurements, a drop of the emulsion was placed between two glass slides and observed with a Leica DM 2500P microscope. Rheological properties of emulsions were characterized with a plate-plate geometry in an AR1000 rheometer (TA Instruments). We eliminate any slip at the plates by coating them with an appropriate sand paper.

\subsection{Synthesis of bulk samples}

Cross-linking of bulk samples was achieved by UV light exposure of fluid cylindrical samples of diameter $35 \mathrm{~mm}$ and thicknesses of 2 or $3 \mathrm{~mm}$. Dymax BlueWave $200 \mathrm{UV}$ lamp was used as a UV source. To avoid water evaporation during the cross-linking process and achieve samples with smooth surfaces, the emulsions were introduced into PTFE molds of various thicknesses and maintained between two quartz plates. The maximum cross-linking (no change in material properties) was achieved after 5 minutes of cross-linking for each side of the sample. After cross-linking, samples were dried in an oven at $60^{\circ} \mathrm{C}$.

\subsection{Synthesis of porous resonators}

Porous resonators were synthesized by UV-cross-linking of droplets constituted of a multiple water/PDMS/glycerol emulsion via a syringe at $2 \mathrm{~mL} / \mathrm{h}$ in the microfluidic co-flow device (see SI-I). The outer phase (glycerol, Fisher Organics) was injected using a pump at pressure of 650 mbar. In order to maintain the uniform structure of the emulsion before cross-linking, magnetic stirring inside the syringe was applied. The cross-linking of beads was triggered by the UV lamp. The length of the exposure channel was about $3 \mathrm{~cm}$, which corresponds to about $3 \mathrm{~s}$ of exposure time. Additional UV exposure had no effect on bead properties. After 
synthesis, the beads were washed with ethanol (97\%, technical grade) and dried in an oven at $60^{\circ} \mathrm{C}$.

\subsection{Characterization of porous structure}

The microstructures of the obtained porous samples, including beads and bulk samples, were characterized with a TM-1000 scanning electron microscope (Hitachi). The macrocellular characteristics were obtained from experiments of mercury intrusion using a Micromeritics Autopore IV 9500 porosimeter with the following parameters: contact angle $=130^{\circ}$, mercury surface tension equal to $485 \mathrm{mN} \mathrm{m}^{-1}$, maximum intrusion pressure equal to $224 \mathrm{MPa}$. For bulk samples, the porosity was also measured by using the mass and the volume of samples. Measurements of the density of the PDMS matrix using AccuPyc 1330 helium pycnometer (Micromeritics) led to a value of $1.04 \mathrm{~g} / \mathrm{cm}^{3}$ for all samples.

In order to characterize the porosity variations inside beads, individual beads of diameter $D$ were immerged in a water-glycerol mixture ( $90 \mathrm{wt} . \%$ of glycerol), and filmed by a camera (Philips SPC530NC). The terminal raising speed $V_{t}$ was used to calculate the density of each bead $\rho_{\text {bead }}$ according to the equation:

$$
\rho_{\text {bead }}=\rho_{\text {medium }}-\frac{18 \eta_{\text {medium }} V_{t}}{g D^{2}}
$$

in which $\rho_{\text {medium }}$ and $\eta_{\text {medium }}$ are respectively the mass density and dynamic viscosity of the water-glycerol mixture and $g$ is the gravitational acceleration.

\subsection{Mechanical properties}

Compression measurements on disk-shaped bulk porous samples were performed using Zwick Z2.5/TN1S apparatus. In the linear elastic regime, the dependence of the normal force $F$ with the strain $\varepsilon$ was obtained and treated according to the Young's law:

$$
\sigma_{N}=E \varepsilon
$$

where $\sigma_{N}=F / S$ is the normal stress, $S$ the sample area and $\varepsilon=\left(h_{0}-h\right) / h_{0}$ with $h_{0}$ and $h$ the sample's thickness before and during compression respectively.

The shear modulus of bulk samples was determined using a Dynamic Mechanical Analysis apparatus (DMA/SDTA861e, Mettler Toledo). To do so, two equivalent samples with diameter $\mathrm{d}=10 \mathrm{~mm}$ were cut from the original disk-like samples and submitted to shear oscillations with frequency varied from 0.01 to $200 \mathrm{~Hz}$ and shear strain amplitudes $\gamma_{\max } \sim$ 0.01 , which is in the linear domain. The shear stress $\sigma_{\tau}$ was registered as function of applied shear strain $\gamma$. This permits to determine the real and imaginary parts of the shear modulus $G$ :

$$
\sigma_{\tau}=\left(G^{\prime}+i G^{\prime \prime}\right) \gamma
$$

Compression tests on individual beads were performed using a rheometer (DHR-2, TA instruments) equipped with a plate-plate geometry. The typical roughness of the plates was 
always much lower than the bead's diameter. The dependence of the normal force with the gap $H$ between the rheometer's plates was obtained and analyzed, using Hertz's equation ${ }^{24}$ :

$$
F=\frac{E \cdot D^{1 / 2}}{3\left(1-v^{2}\right)}(D-H)^{3 / 2}
$$

where $F$ is the normal force, $D$ the diameter of the bead, $E$ the bead's Young's modulus and $v$ the Poisson's ratio. Using the effective modulus $E^{*}=E /\left(1-v^{2}\right)$ and displacement $\delta=D-H$, one obtains:

$$
\frac{F}{D^{2}}=\frac{E^{*}}{3}\left(\frac{\delta}{D}\right)^{3 / 2}
$$

Equation (5) allows comparison of mechanical properties of beads with different diameters. Average moduli were obtained from data on about 20-30 beads for each sample.

\subsection{Acoustical measurements}

The sound speed in disk-shaped bulk porous materials was obtained from direct contact measurements on two samples with 2 and $3 \mathrm{~mm}$ in thickness and $35 \mathrm{~mm}$ in diameter as previously done ${ }^{9}$. Each sample was placed between two identical broadband ultrasonic (US) transducers (emitter and receiver, Olympus V301) with a diameter of $30 \mathrm{~mm}$ and a central frequency of $500 \mathrm{kHz}$. The emitting transducer was excited with short (broadband) pulses generated by a pulser/receiver (Olympus, 5077PR-40-E) that was also used to amplify the electric signal recorded by the receiving transducer before its acquisition on a computer via an oscilloscope (Lecroy, 9450A).

In order to test the resonant properties of beads, the latest were dispersed in a $0.2 \% \mathrm{wt}$. aqueous gel (Carbopol ETD 2050, Lubrizol). The suspensions of beads had a volume concentration equal to $5 \%$. The needed mass of beads was calculated using previously obtained average density value. To prevent any bubble formation or trapping, the mixing of the beads with the gel was done using a fritted glass filter connected to a syringe (see the image in the SI). The beads were weighted on the filter and covered by a significant amount of a bubbles-free gel. Because of the hydrophobic properties of PDMS, the gel does not penetrate between the beads. The immersion of beads in the gel was achieved by gently evacuating the remaining air bubbles using a syringe connected to a needle. Once trapped in the gel, the beads were transferred to a plastic vial and diluted with the rest of the gel under slow mixing.

The suspension of the resonators in the gel was transferred to a $100 \mathrm{~mm} \times 100 \mathrm{~mm} \times 1.2 \mathrm{~mm}$ cell with parallel sides for the acoustical measurement. The cell was immerged into a water tank and placed between two transducers (the same pair as used previously for the characterization of bulk samples). Then, the transmission factor was obtained by calculating the ratio of the Fourier transforms of the temporal signals acquired without and with the measurement cell in the path of the acoustic beam. 


\section{Results and discussion}

\subsection{Emulsion properties}

In order to control the structure of porous silicone materials, we synthesized them by crosslinking water/PMDS emulsions with similar size of droplets, but different interactions between droplets. Such interactions were tuned by using two different surfactants: a) Silube, which is not fully soluble in water and PDMS, at a concentration of $0.4 \%$ by weight with respect to the continuous phase, and b) $\mathrm{OH} 457$, which is fully miscible with PDMS, but not with water, at a concentration of $0.4 \%$ by weight with respect to the continuous phase. Despite the fact that Silube is not fully miscible with neither of the two phases, it was nevertheless possible to emulsify water in the PDMS/Silube inhomogeneous mixture. The emulsions, prepared by mechanical stirring in similar conditions as described in the experimental section, were characterized before cross-linking of the PDMS continuous phase.

Figure 1 shows photographs of the emulsions obtained with the optical microscope. For better visualization, emulsions were diluted with PDMS to obtain 5\% vol. concentration of the aqueous phase. In both samples one observes spherical water droplets with diameters ranging from 1 to $10 \mu \mathrm{m}$. Water droplets in the emulsion with Silube are aggregated (Figure 1a) whereas the droplets of the OH457-stabilized emulsion remain fully dispersed and nonaggregated in the continuous phase (Figure 1b).
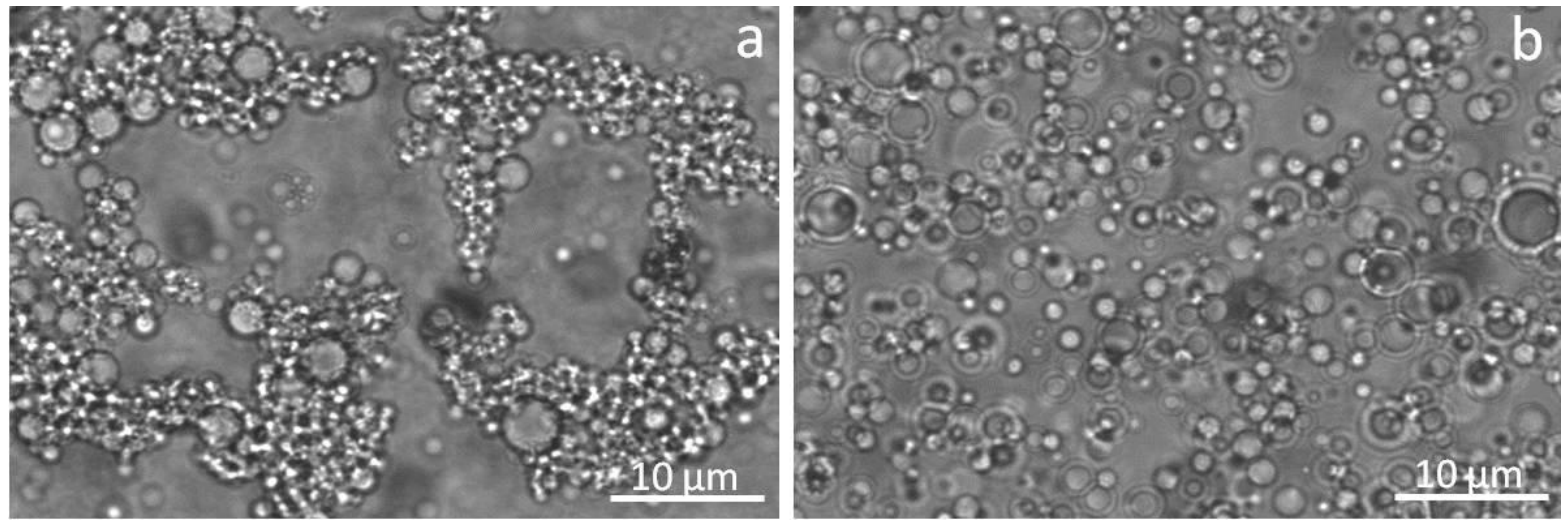

Figure 1-Optical images of water/PDMS emulsions (5\% vol. aqueous phase) stabilized with Silube (a) and OH457 (b) surfactant. The emulsions were obtained dilution with PDMS of initially stabilized $30 \%$ vol. emulsions containing $0.4 \% \mathrm{wt}$. surfactant.

The size distributions of these two emulsions obtained from static light scattering are presented on Figure 2a. In order to prevent droplet aggregation, the measurements were performed on emulsions diluted in dodecane. Optical microscopy observations indeed show that both emulsions are fully dispersible in dodecane (not shown here). The graphs show roughly similar size distributions and only slightly different average diameters $(\sim 4 \mu \mathrm{m}$ for Silube and $\sim 10 \mu \mathrm{m}$ for $\mathrm{OH} 457$ ) for both emulsions. 

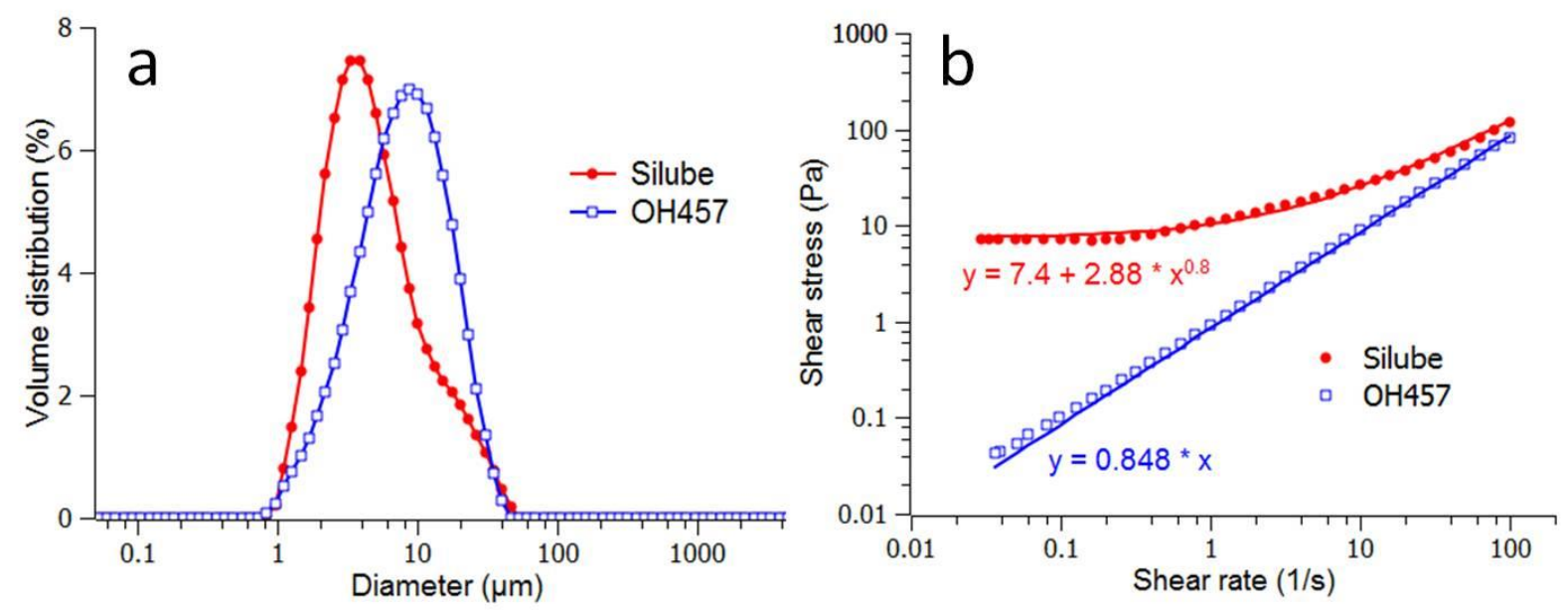

Figure $2-a)$ Size distribution of water droplets in the emulsion stabilized by Silube (filled circles - -) and OH457 (open circles -0-) after their dilution in dodecane and as obtained by static light scattering; b) Variation of steady shear stress with shear rate for water/PDMS emulsions $(30 \%$ wt.) obtained with Silube (०) and OH457 (•) surfactants stabilized emulsions; surfactant concentrations: $0.4 \%$. The solid lines represent the results of the fit to a corresponding model (see in the text).

However, as shown on the Figure $2 b$, the two emulsions exhibit very different rheological behaviors, which is due to the different interactions between droplets and consequently different structures of the emulsions. In the OH457-stabilized emulsion, we observe a Newtonian behavior, which is well fitted by the Newton's law $\tau=\eta \dot{\gamma}$, where $\eta$ is the viscosity and $\dot{\gamma}$ the shear rate. The emulsion stabilized with Silube exhibits a pronounced yield stress behavior which is due to the presence of the droplets aggregates that percolate in the PDMS continuous phase (as seen in Figure 1a). The data can be fitted correctly to the Hershnell-Buckley model $\tau=\tau_{0}+k \dot{\gamma}^{n}$, in which $\tau_{o}$ is the yield stress, $k$ is the consistency and $n$ is the flow index.

Finally, the structures of the emulsions obtained in this study are well-defined and present two limit cases which are possible in the system: i) a fully aggregated structure as droplets attract each other due to the poor solvent quality of PDMS versus Silube; ii) a fully dispersed structure as droplets repel each other with $\mathrm{OH} 457$.

\subsection{Porous structure of bulk materials}

We used emulsions with interconnected and isolated droplets to obtain bulk materials. After cross-linking and drying, samples have different appearance as illustrated by Figure 3 in comparison with a non-porous sample obtained without emulsion. While Silube sample is opaque as expected for a macroporous material, the $\mathrm{OH} 457$ sample is transparent, similarly to the non-porous one. 

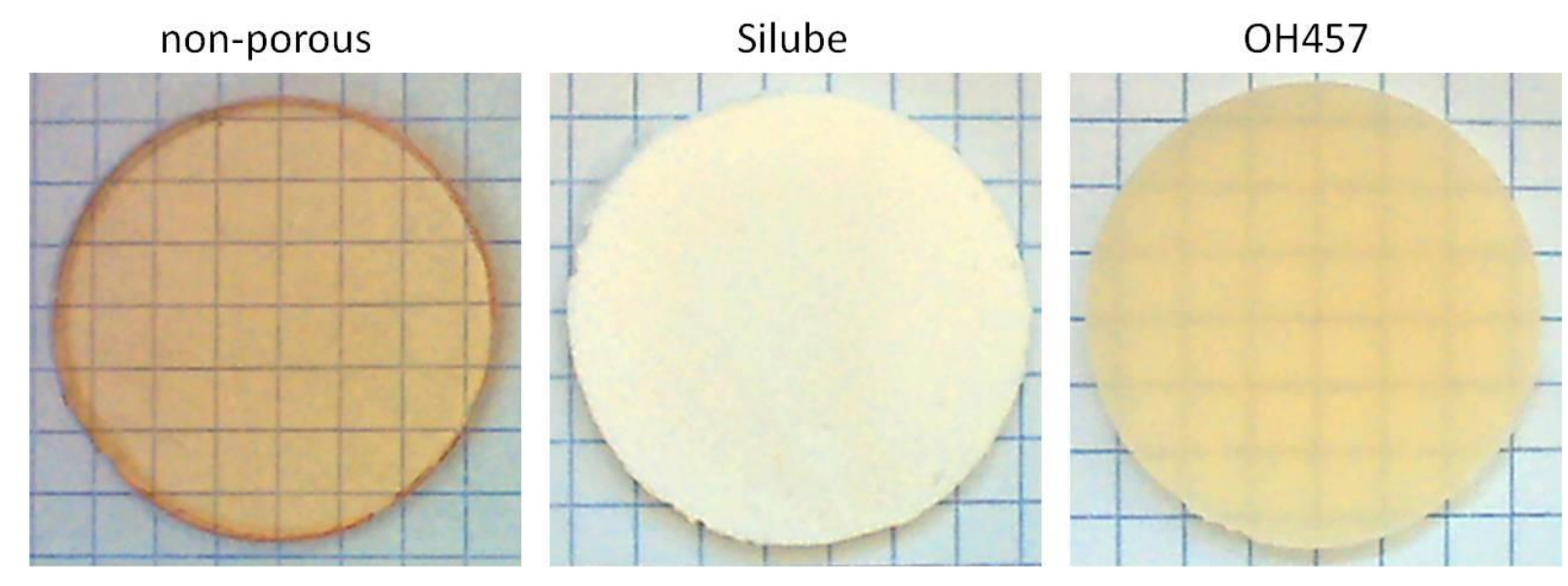

Figure 3 - Photographs of dry bulk samples prepared by cross-linking pure PDMS or water/PDMS emulsion stabilized by Silube or OH457 surfactant (image width $=4 \mathrm{~cm}$ ).

In order to explain this difference, we have analyzed the porous structure of the samples by mercury intrusion/extrusion and scanning electron microscopy (SEM). Mercury intrusion curves and corresponding interconnection size distributions are shown on Figure 4. For all samples, rising of the intrusion line is observed at high pressures. This rising is reversible when lowering the pressure and corresponds to bulk compression of the matrix. As expected, the non-porous PDMS does not show any macroporosity. For Silube sample, porosity is equal to the initial water volume fraction (30\%) and average pore interconnection size is about $5 \mu \mathrm{m}$ which is coherent with initial water/PDMS emulsion size distribution. Surprisingly, intrusion curves of the OH457 sample are similar to the non-porous PDMS which indicates the collapse of the macroporous structure.
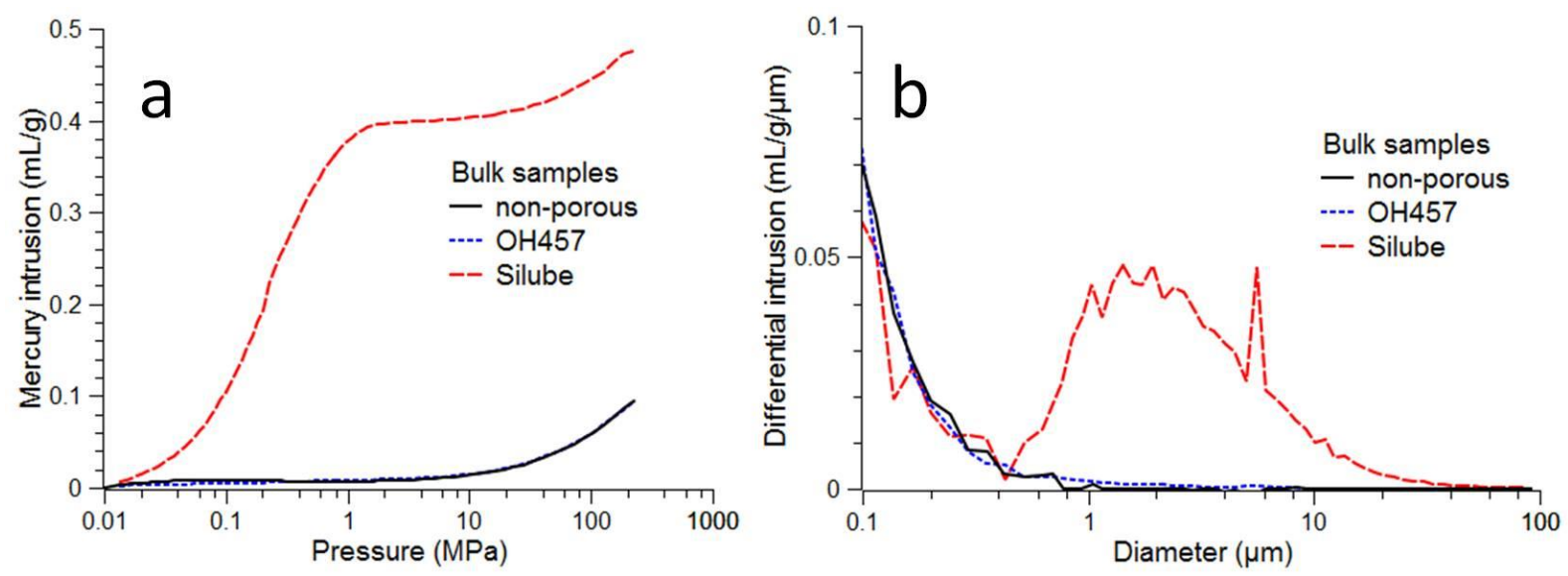

Figure 4 - Mercury intrusion curves (a) and pore interconnection size distributions (b) of bulk samples. The non-zero intrusion at high pressures (corresponding to low diameter values) is due to bulk compression of the PDMS matrix.

SEM images (Figure 5) are coherent with the porosimetry data. The Silube sample has an interconnected system of pores. The pores are organized in channels and have diameters ranging from 1 to $15 \mu \mathrm{m}$. Thus, the pore structure is similar to the morphology of droplets inside Silube-stabilized emulsion. By contrast, pores inside OH457 sample are completely collapsed and difficult to observe with SEM. 

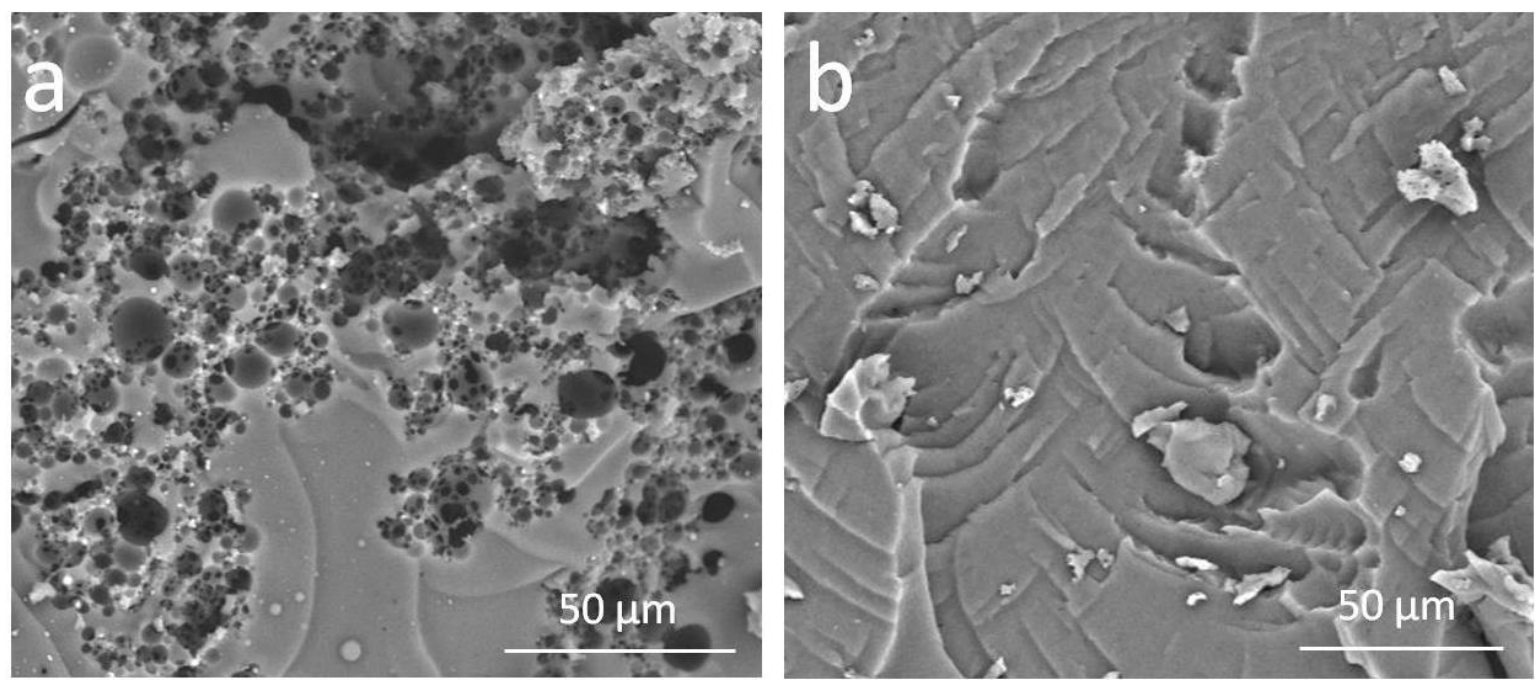

Figure 5 - Scanning electron microscopy (SEM) images of dry bulk samples obtained with Silube (a) and $\mathrm{OH} 457$ (b) surfactant.

\subsection{Mechanical and acoustical properties of bulk materials}

Mechanical characterization was done on 4 equivalent disk-like samples in the Zwick apparatus. Typical compression curves are plotted on Figure 6a. For deformations less than 0.02 , the observed deviation of the curves from linearity corresponds to the pre-contact region in which the non-parallelism and the roughness of the surfaces affect the values of the measured normal stress. Non-porous PDMS shows linear stress-strain curve at strain values less than 0.1 and further fractures under compression. This behavior is uncommon for elastomers and is due to the epoxy-modified molecular structure of UV-cross-linkable PDMS. The fit to eq (2) leads to a Young's modulus values $E$ around 7.3 MPa. Similar curve is observed for the collapsed OH457 sample. By contrast, the stress-strain curve of the Silube sample contains two regions. The first region $(\varepsilon<0.2)$ demonstrates linear behavior with $E$ around 1.2 MPa. At higher deformations, densification of the sample leads to the higher modulus. Our observations clearly show the correlation of the mechanical properties with the porous structure of the samples. 

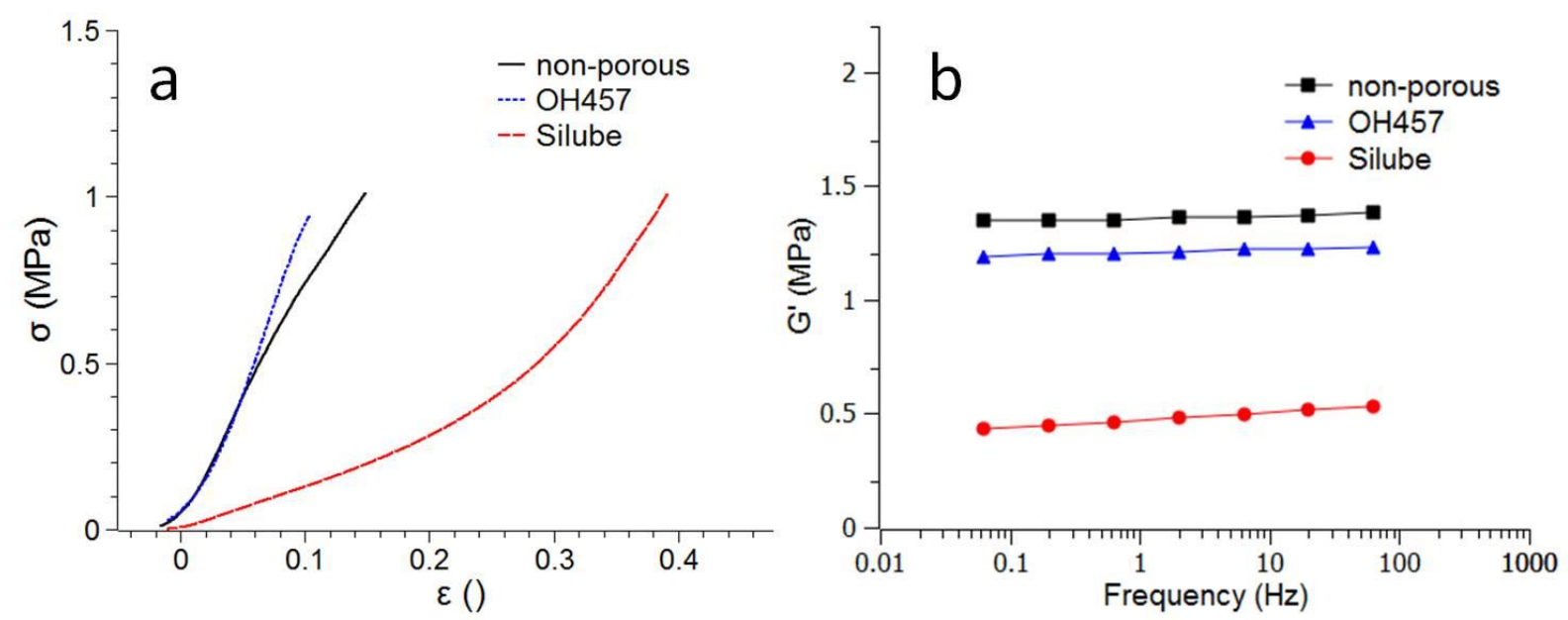

Figure 6 - Mechanical properties of bulk silicone samples prepared using Silube or OH457stabilized emulsions in comparison with that of a non-porous sample: a) strain-stress compression curves; $b$ ) real part of the shear modulus as a function of frequency.

Dynamic mechanical analysis (DMA) curves of bulk samples in shear geometry are presented on the Figure $6 \mathrm{~b}$. The real part of the shear modulus $\left(G^{\prime}\right)$ doesn't show frequency dependence in the $0.1-100 \mathrm{~Hz}$ frequency range (a). $G$ ' values of non-porous and porous $\mathrm{OH} 457$ samples are similar (1.35 and 1.2 MPa, respectively) while the value of Silube sample is significantly lower $(0.45 \mathrm{MPa})$. This correlates with the porous structure of the samples. For any material, Young and shear moduli are linked by the Poisson's ratiov.

$$
G=\frac{E}{2(1+v)}
$$

In pure elastomers, it is well established that the Poisson's ratio $v$ is very close to 0.5 . However, the expected values of the shear modulus for the non-porous and $\mathrm{OH} 457$ samples using eq. 6 are equal to $2.4 \mathrm{MPa}$. The measured values of $G$ ' are lower than the expected ones which may be explained by the large measurement uncertainties as reported in the literature ${ }^{25}$.

Using again eq (6), one can estimate the Poisson's ratio of the porous Silube material: $v \sim 0.33$. This value of $v$ is in good agreement with both theoretical predictions and experimental results obtained for porous materials. 1

The longitudinal sound speed values $\left(C_{L}\right)$ in the bulk samples is also a direct function of the mechanical modulus through the following equation:

$$
C_{L}=\sqrt{\frac{K+\frac{4}{3} G}{\rho}}
$$

where $\rho$ is the materials mass density and $K$ the bulk modulus. The experimental values of $C_{\mathrm{L}}$ are listed in Table 1. The sound speed in the Silube sample is very low, about $70 \mathrm{~m} / \mathrm{s}$ as previously observed ${ }^{9,12}$, in contrast with the value of $\sim 1000 \mathrm{~m} / \mathrm{s}$ measured in the OH457 sample, which is almost the same as in non-porous PDMS. 
Thus, we observe the correlation between mechanical and acoustical properties. The low value of the speed inside the Silube sample is due to the compressible nature of the porous materials and correlates with the value of the bulk modulus $K$ :

$$
K=\frac{G}{3} \cdot \frac{1+v}{0.5-v}
$$

As expected from eq (8), the value of $K$ is drastically decreased as the Poisson's ratio drops down from about 0.5 to 0.33 . Collapsed $\mathrm{OH} 457$ sample has the mechanical properties similar to the non-porous PDMS and thus has high $K$ and $C_{L}$ values.

Table 1 - Measured values of the acoustical and mechanical parameters of porous silicone samples.

\begin{tabular}{|c|c|c|c|c|c|c|c|}
\hline & \multicolumn{3}{|c|}{ Bulk samples } & & \multicolumn{3}{|c|}{ Resonators (beads) } \\
\hline & Non-porous & Silube & OH457 & & Non-porous & Silube & OH457 \\
\hline$\Phi(\%)$ & 0 & 30 & 0 & $\Phi(\%)$ & 0 & 25 & $\approx 0$ \\
\hline$\left\langle d_{c}>(\mu m)\right.$ & - & 4.9 & - & $\left\langle d_{c}>(\mu m)\right.$ & - & 6.2 & \\
\hline$C_{L}(\mathrm{~m} / \mathrm{s})$ & 1100 & 70 & 1000 & $C_{L}(\mathrm{~m} / \mathrm{s})$ & $1100^{+}$ & $120^{+}$ & $1000^{+}$ \\
\hline$E(M P a)$ & 7.3 & 1.2 & 7.3 & $E^{*}(M P a)$ & 6 & 2 & 3.8 \\
\hline$G^{\prime}(M P a)$ & 1.35 & 0.45 & 1.2 & & & & \\
\hline
\end{tabular}

+ values that fit well (solid line) to the experimental spectra shown in Figure 11.

\subsection{Visualization of the pore drying}

As shown in the previous section, interconnected porous structure is necessary for obtaining air-filled silicone materials. The connections between pores are crucial for the replacement of water by the air, which was visualized in the following experiment. A thin layer (about 0.05 $\mathrm{mm}$ ) of emulsion stabilized with Silube or OH457 surfactant was cross-linked between two quartz slides. One of the sides of the sample was left non-protected in order to induce water evaporation. The sample was put in a thermally controlled Peltier microscope cell. The drying temperature was equal to $25^{\circ} \mathrm{C}$ and $70{ }^{\circ} \mathrm{C}$ for Silube and $\mathrm{OH} 457$ sample, respectively. Due to the difference in the refractive indices of PDMS, water and air, ${ }^{26}$ the drying front can be clearly observed. Figure 7 presents images of the drying front at two different times (time difference is about $30 \mathrm{~min}$ ) for the samples with Silube and OH457. In the case of Silube (Figure $7 \mathrm{a}$ and $\mathrm{b}$ ), the percolated network of pores is clearly observed and the drying leads to the instantaneous replacement of the liquid water that is initially present inside the pores (thus exhibiting a low optical contrast with the PDMS matrix) by air (that exhibits a much higher optical contrast with PDMS). One can guess that the PDMS matrix is subjected to almost negligible mechanical stress since the exchange through the air-water interface is favored due to the relatively large size of the connections (typically $\sim 1 \mu \mathrm{m}$ from the SEM photograph of Figure 5c, i.e. a size much larger than the molecular scale) between the pores. No change in the porous structure is observed during this process. By contrast, in the case of $\mathrm{OH} 457$ (Figure $7 \mathrm{c}$ and $\mathrm{d}$ ), water-filled pores are homogeneously distributed, with no interconnections, in the sample and disappear after drying, yielding a transparent PDMS. From the SEM pictures of the dried beads (d-f) and bulk PDMS samples (see SI), one clearly observes the collapse of 
the pores. This is similar to the collapse of cross-linked porous hydrogels during drying ${ }^{27}$ : because of the high permeability of the matrix (both hydrogel and $\mathrm{PDMS}^{28}$ ), water molecules are diffusing from the inner droplets outside the material and the pressure inside the pores decreases and can attain negative values. If the matrix is stiff (as in highly cross-linked hydrogel, for example ${ }^{27}$ ), the water inside pore becomes mechanically unstable and a vapor cavity appears. The threshold pressure of the cavitation is about $22 \mathrm{MPa}{ }^{27,29}$ However, when the matrix is soft (weekly cross-linked hydrogel ${ }^{27}$ or PDMS in the present paper), stress relaxation may take placeREF. Therefore, we suppose that stresses occurring locally inside the PDMS matrix near the pore during drying may lead to the irreversible pore collapse. In our case, the stress is much higher in the case of the closed pores system $(\mathrm{OH} 457)$ compared to the open-pore one (Silube), in which the air can penetrate in the material by the connected channels of pores. Furthermore, adhesion forces between pore walls can contribute to maintain the collapse state, similarly to the so-called "stiction" effect (sticking of surfaces after a capillary induced deformation) which is well documented in the case of MEMs (Micro-Electro-Mechanical devices) ${ }^{30}$.
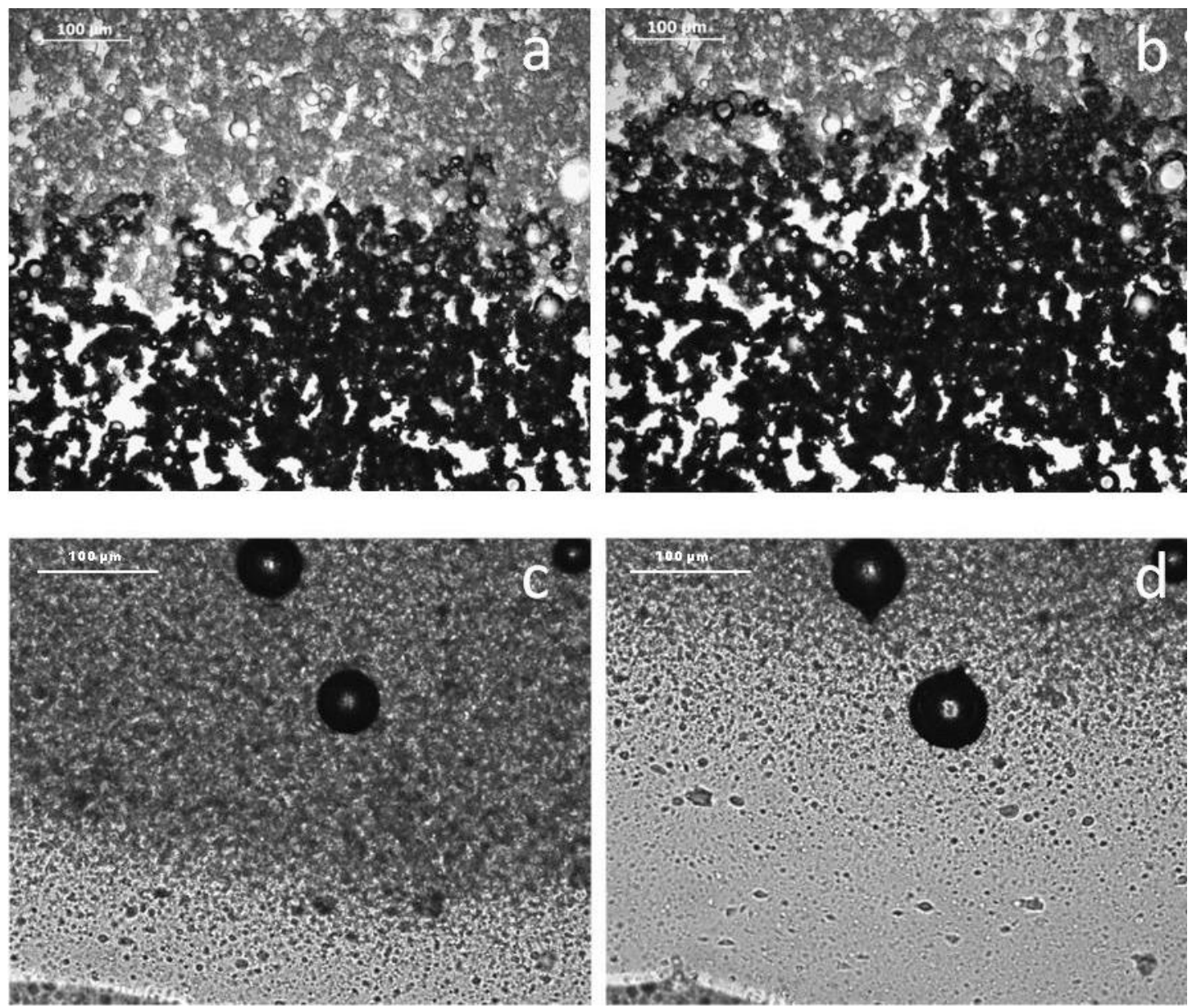

Figure 7 - In-situ optical microscopy observations of the drying front of thin porous silicone layers synthesized using emulsions stabilized by Silube $(a$ and $b)$ or OH457 ( $c$ and $d)$. The drying is driven from the edge of the sample situated at the bottom of the image. The temperatures of the drying are $20^{\circ} \mathrm{C}\left(a\right.$ and $b$ ) and $70^{\circ} \mathrm{C}(\mathrm{c}$ and $d)$. Images $b$ and $d$ show the same regions as the images $a$ and $c$, respectively, after 30 min of observation. 


\subsection{Porous structure of resonators}

We have also studied the porous structure and properties of beads produced in microfluidic device using Silube or OH457-stabilized emulsions.

Figure 8 (a-c) shows the SEM images of porous resonators, synthesized from Silubestabilized emulsion in the co-flow device. The beads have a spherical shape and an opened porosity. Pores are also observed at the surface of the beads (Figure 8a). From the crosssection image (Figure $8 b$ and $c$ ), one can see that spherical pores inside the beads form aggregates crossing the volume and coming out to the surface. The sizes of the cells inside the aggregates are roughly comprised between 1 and $10 \mu \mathrm{m}$, which correspond to the sizes of water droplets in the initial emulsion.

The morphology of the porous materials obtained from the OH457-stabilized emulsion is completely different from the one of Silube. Figure 8(d-f) presents some SEM images of the resonators obtained with $\mathrm{OH} 457$ after drying. The beads have invaginations on their surface and their shapes are distorted from the spherical one (Figure 8d). The pores which are seen on the cross-section (Figure 8e and 4f) are isolated and have a lentil-like shape. These observations correlate with the observations on the bulk samples and confirm the collapse of the porous structure of $\mathrm{OH} 457$ sample during drying which leads to a much denser material as compared to the Silube one.
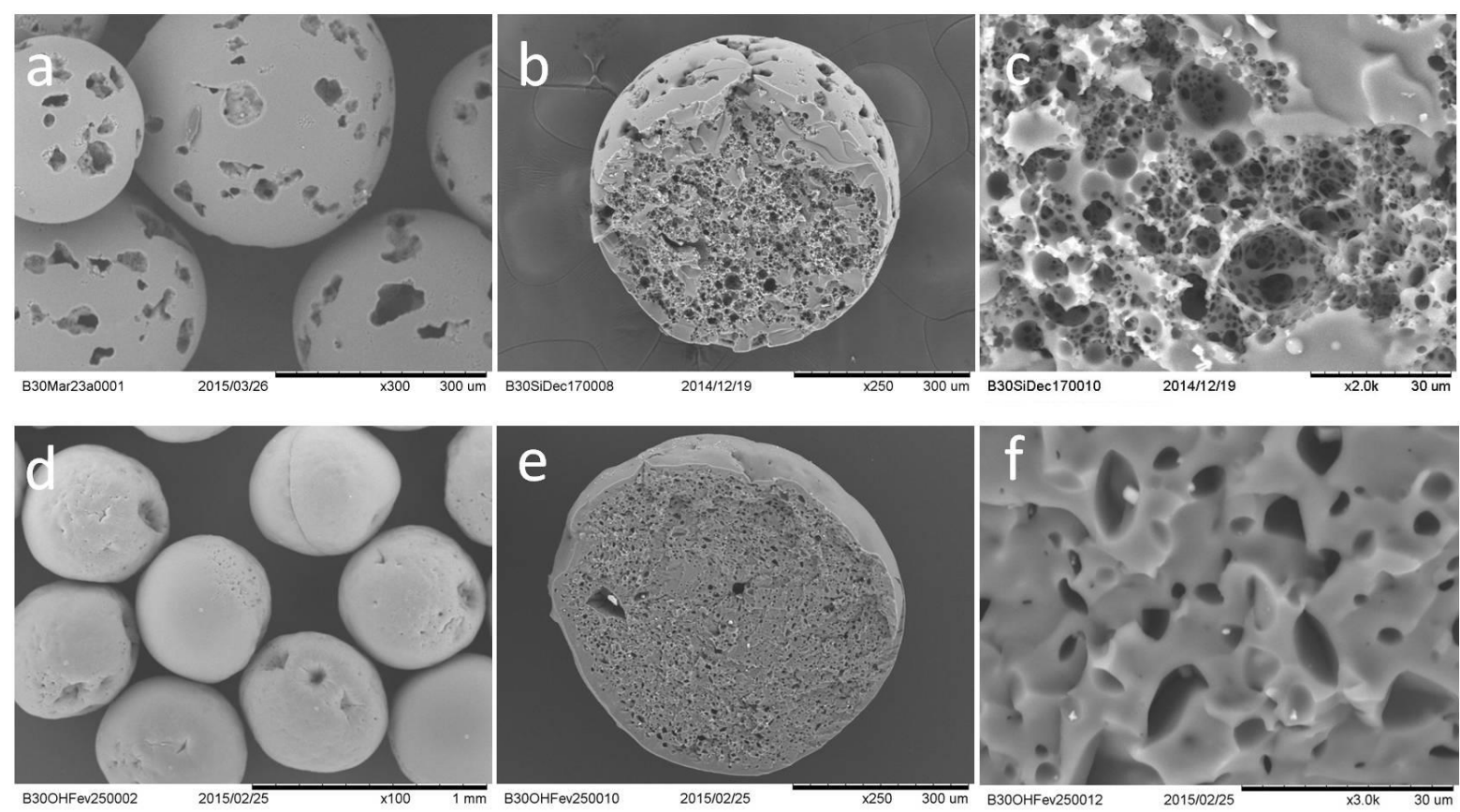

Figure 8 - Scanning electron microscopy (SEM) images of dried porous silicone resonators obtained using the emulsion stabilized by Silube $(a-c)$ or OH457 $(d-f)$ : overall view $(a$ and $d)$; cross-section ( $b$ and e); internal structure ( $c$ and $f$ ).

Mercury intrusion curves for beads are provided in the SI-III. The results, summarized in the Table 1, are similar to those of bulk samples. The porosity of the beads produced with Silube 
is slightly lower (25\%) with respect to bulk samples (30\%). This may be explained by loss of water from the droplets inside the microfluidic device. The intrusion curve of the OH457 beads has a slight raise at low pressures (about $0.02 \mathrm{MPa}$ ), which is probably due to the interstitials between beads or to the invaginations on the bead surfaces.

Some changes in the porous structure were observed between different beads by SEM. Thus, we analyzed the flotation speed of about 15 beads of each type in a water-glycerol mixture with a known viscosity. The average density values deduced from the density distributions are plotted in the SI-IV.

The densities of resonators with Silube vary from 600 to $850 \mathrm{~kg} / \mathrm{m}^{3}$ with the average value of $760 \pm 70 \mathrm{~kg} / \mathrm{m}^{3}$, which matches very well the mercury porosimetry data. The variations from the theoretical value of $700 \mathrm{~kg} / \mathrm{m}^{3}$ are due to the inhomogeneous aggregated structure of the emulsion used to synthesize the beads: during the formation and rupture of the microfluidic jet with aggregated water droplets, the amount of water varies significantly from one water/PDMS/glycerol droplet to another.

The calculated densities of resonators from OH457-stabilized emulsion are much higher than the theoretical ones and are close to those of pure PDMS. Taking into account the SEM observations, we can conclude that the pores in this type of resonators are shrunk.

The difference between internal structures of the resonators with Silube and OH457 is clearly seen on the images of optical microscopy of beads in $0.2 \%$ Carbopol gel, presented on Figure 9. In the case of Silube (Figure 9a), the pores inside the resonators exhibit a high optical contrast in the bright field due to the efficient light diffusion at the PDMS/air interface. Squeezing of the beads between two microscope slides leads to the appearance of air bubbles on their surface (Figure 9b). Resonators synthesized from OH457-stabilized emulsion have a homogeneous and transparent structure (Figure 9c). Destruction of these beads by squeezing (Figure 9d) shows the absence of air inside them (except few surface bubbles that were initially formed) which confirms that all pores in the PDMS were almost fully collapsed.
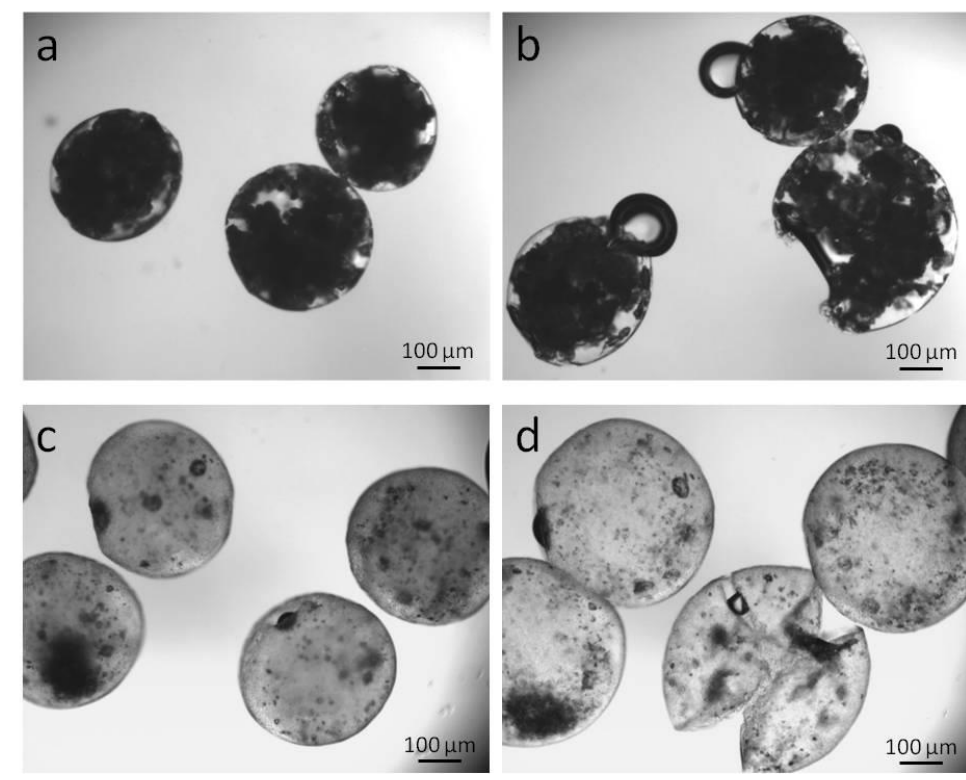
Figure 9 - Optical microscopy images of beads synthesized using emulsions stabilized by Silube ( $a$ and $b$ ) or $\mathrm{OH} 457$ ( $c$ and $d$ ). The images are taken from beads immerged in $0.2 \%$ Carbopol gel before ( $a$ and $c)$ and after $(b$ and $d$ ) squeezing between the microscope glass slides.

\subsection{Mechanical and acoustical properties of resonators}

Mechanical properties of the resonators were studied by submitting them to a compression between two parallel plates of the rheometer. Figure 10a shows the compression curve in the coordinates corresponding to the eq (4). For all types of beads, including non-porous beads and porous beads prepared using Silube or $\mathrm{OH} 457$, the ratio $F / D^{2}$ scales linearly with $(\delta / D)^{3 / 2}$ as predicted by eq (3) in the limit of small deformation, which we observe to be valid in our case up to $F / D^{2} \sim 0.1 \mathrm{MPa}$ and $\delta / D \sim 0.2$. Fitting our data to eq (3), one gets the histograms of the effective modulus values for three types of beads on Figure 10b. The non-porous beads have the narrowest average effective modulus around $6 \mathrm{MPa}$. The values of $E^{*}$ for porous resonators are lower, the mean values for Silube and $\mathrm{OH} 457$ being equal to $2 \mathrm{MPa}$ and 3.8 $\mathrm{MPa}$, respectively. As described in the previous sections, the sample with $\mathrm{OH} 457$ has collapsed pores and the density close to that of the non-porous PDMS. We believe that the lower effective modulus of the beads with $\mathrm{OH} 457$ with respect to the non-porous beads can be explained by the lower degree of cross-linking in the beads prepared using the emulsion. We have made an attempt to estimate this degree of cross-linking from the glass transition temperature $\left(T_{\mathrm{g}}\right)$ shift, which is reported in the SI-V. Other methods as swelling in organic solvents and IR spectroscopy of the epoxy band did not give reliable results because of the porous nature of the samples and strong silicone absorption bands, respectively. The $T_{\mathrm{g} S}$ of the samples prepared from water/PDMS emulsions were significantly lower than that of pure cross-linked PDMS and close to the $T_{g}$ of liquid PDMS before cross-linking. Thus, this indicates that the degree of cross-linking of the porous samples is lower than of the nonporous ones, which may be due to a suppression of the cross-linking reaction by reaction of the oxonium ions with water molecules ${ }^{31}$. The $T_{\mathrm{gS}}$ of the porous Silube and OH457 samples are similar, which means that the cross-linking degrees of these samples are also similar. However, the values of the effective modulus $E^{*}$ of the Silube resonators are significantly lower, which is due to their air-filled porous structure.
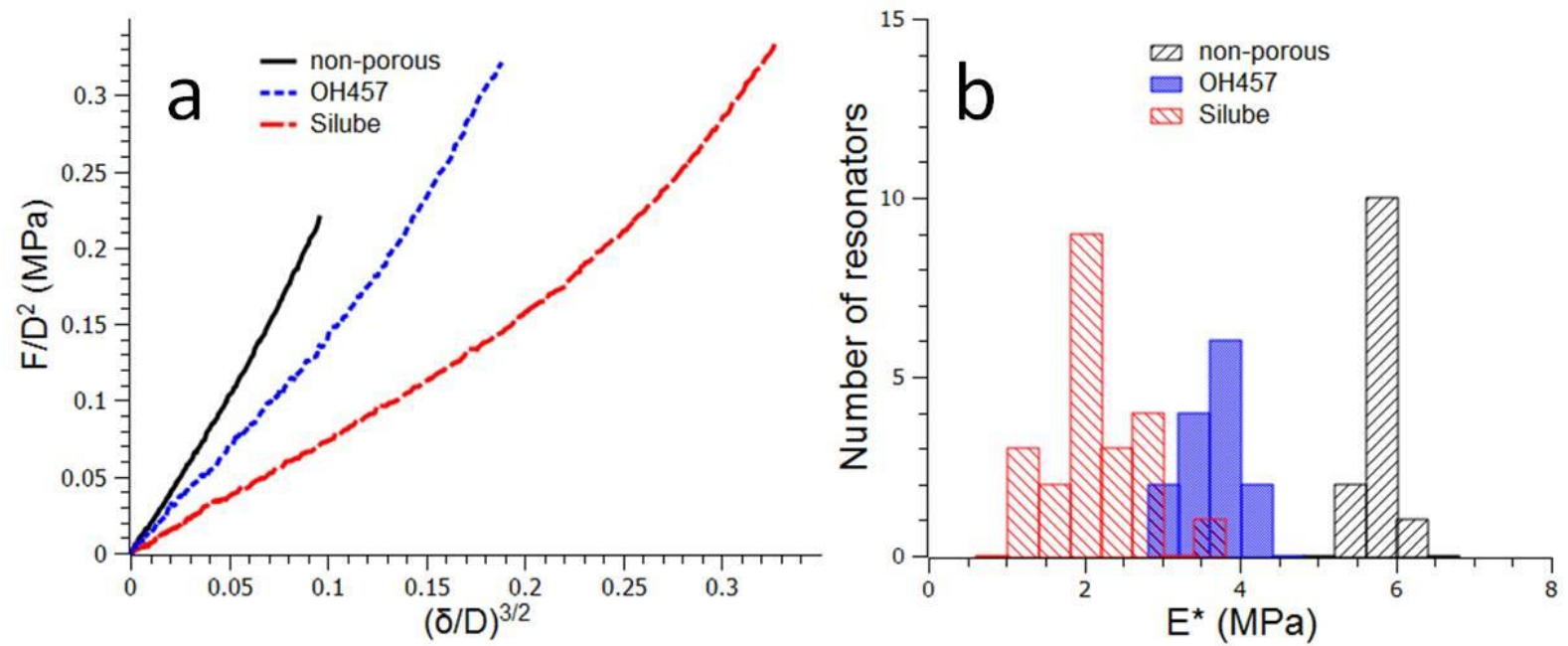
Figure 10 - Mechanical properties of silicone resonators with isolated pores (OH457) and interconnected pores (Silube) in comparison with non-porous beads: a) typical dependence of the ratio $F / D^{2}$ with the displacement $\left.\delta ; b\right)$ histogram of the effective mechanical modulus $E^{*}$ obtained from the linear fit of the curves at small deformations to eq (5) in the text.

The acoustic transmission spectra of resonators, dispersed in a $0.2 \% \mathrm{wt}$. aqueous gel at the volume concentration of $5 \%$, are presented on Figure 11. We observe a pronounced minimum at about $0.2 \mathrm{MHz}$ on the experimental spectrum of the Silube beads sample, which is due to the Mie resonance of the ultra-slow beads.

Using the size distribution of the beads and assuming the internal sound velocity $C_{L}$ to be close to $100 \mathrm{~m} / \mathrm{s}$, we modeled the transmission spectrum, and the resulted curve (the solid line on the Figure 11) fits well to the experimental data with $C_{\mathrm{L}}=120 \mathrm{~m} / \mathrm{s}$ (cf Table 1).

The transmission of the $\mathrm{OH} 457$ sample is close to 1 for all frequencies ranging from 0.1 to 0.8 MHz. The beads have poor resonant properties because of the collapsed porosity. As shown in the previous sections, the collapse of pores decreases the air volume fraction to few percents, which leads to much higher bulk mechanical modulus. The value of $C_{L}$ in such collapsed porous beads is difficult to estimate. However, the overlapping of the transmission spectra without any resonance, obtained for the non-porous beads (not shown here) and the collapsed porous beads (blue line in Figure 13) suggests that the sound speeds within these two types of beads are approximately equal $(\sim 1000 \mathrm{~m} / \mathrm{s})$.

The observed difference of the values for the Silube samples in bulk form and in form of resonators can be explained by the fact that the diameter of beads (about $300 \mu \mathrm{m}$ ) is comparable to the typical size of emulsion droplet aggregates (30-50 $\mu \mathrm{m})$, and consequently, to the typical size of the pore clusters. This induces variations in the porous structure inside each bead and between different beads, and leads to a large dispersion of the porosity and mechanical modules of the beads. 


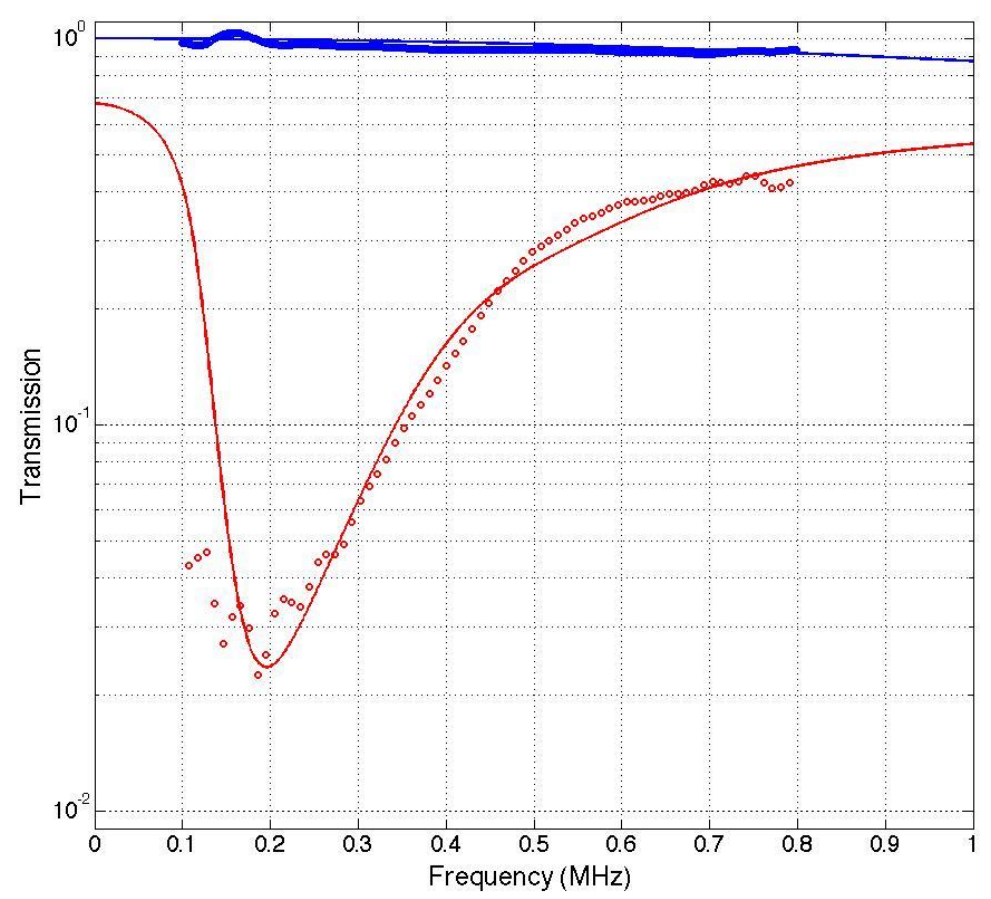

Figure 11 - Ultrasound transmission spectra of samples containing resonators with interconnected (Silube, red circles) or isolated (OH, blue circles) pores. Solid lines: predictions of Multiple Scattering Theory.

\section{Conclusion}

We have shown that mechanical and acoustical properties of porous silicones, produced by cross-linking of water-PDMS emulsions (polyHIPE method), are strongly affected by the emulsion structure. Using a surfactant which is either low or highly soluble in the PDMS phase, we produced emulsions with aggregated or isolated droplets, respectively. Due to this structure difference, the two systems exhibit very different rheological properties. Starting from these two emulsions, we elaborated bulk samples, as well as spherical beads in a co-flow device. In case of aggregated emulsion, the pores of the cross-linked material formed a percolated network in which air easily penetrates during drying, leading to a highly porous structure with low mechanical moduli and sound speed. In the case of non-aggregated emulsion, the pores in the cross-linked silicone had no connections (windows) between them, and the material collapsed completely and irreversibly during drying. Our results demonstrate the importance of interconnections between water droplets for preserving the structure of soft porous materials during drying. When this condition is ignored, complete collapse of the porous structure can lead to the dramatic change in mechanical and acoustical properties.

\section{Author Information}

Corresponding authors:

* E-mail: kovalenko@crpp-bordeaux.cnrs.fr 
*E-mail: mondain@crpp-bordeaux.cnrs.fr

Notes :

The authors declare no competing financial interest.

\section{Acknowledgments}

We thank Guillaume Pibre and Tania Ireland from Bluestar Silicones Company for fruitful discussions and for providing us with silicone rubber and Joël Valencony from Siltech Company for the surfactants. We also thank Ludovic Dufourg for his help in the acoustic characterization of bulk samples and Marc Birot for mercury porosimetry measurements. Fruitful conversations with Christian Frétigny, Annie Colin, Jacques Leng and Olivier Poncelet are gratefully acknowledged. This work was partially funded and performed within the framework of the Labex AMADEUS ANR-10-LABEX-0042-AMADEUS with the help of the French state Initiative d'Excellence IdEx ANR-10-IDEX-003-02. 


\section{References:}

${ }^{1}$ Gibson, L. J.; Ashby, M. F. Cellular Solids: Structure and Properties, 2nd ed.; Cambridge University Press: Cambridge, 1997.

${ }^{2}$ Ren, J.; Langmi, H. W.; North, B. C.; Mathe, M. Review on processing of metal-organic framework (MOF) materials towards system integration for hydrogen storage. Int. J. Energy Res., 2015, 39, 607-620.

${ }^{3}$ Combined and Hybrid Adsorbents, Fundamentals and Applications, NATO Security through Science Series C: Environmental Security, Loureiro, J. M., Kartel, M.T., Eds.; Springer, Dordrecht, 2006.

${ }^{4}$ Hierarchically Structured Porous Materials: From Nanoscience to Catalysis, Separation, Optics, Energy, and Life Science, ed. Su, B. L.; Sanchez, C.; Yang, X. Y., Eds.; Wiley-VCH Verlag GmbH \& Co. KGaA, Weinheim, Germany. doi: 10.1002/9783527639588.ch10, 2012.

5 Sopyan, I.; Mel, M.; Ramesh S.; Khalid K.A. Porous hydroxyapatite for artificial bone applications. Sci. Technol. Adv. Mater. 2007, 8, 116.

${ }^{6}$ Zellander, A.; Zhao, C.; Kotecha, M.; Gemeinhart, R.; Wardlow, M.; Abiade, J.; Cho, M. Characterization of pore structure in biologically functional poly(2-hydroxyethyl methacrylate) - poly(ethylene glycol) diacrylate (PHEMA-PEGDA). PLOS ONE, 2014, 9, 1-8.: e96709. doi:10.1371/journal.pone.0096709

${ }^{7}$ Attenborough, K. Acoustical characteristics of porous materials. Phys. Rep. 1982, 82, 179.

${ }^{8}$ Gross, J.; Fricke, J.; Hrubesh, L.W. Sound propagation in $\mathrm{SiO}_{2}$ aerogels. J. Acoust. Soc. Am. 1992, 91, 2004-2006.

${ }^{9}$ Zimny, K.; Merlin, A.; Ba, A.; Aristégui, C.; Brunet, T.; Mondain-Monval, O. Soft porous silicone rubbers as key elements for the realization of acoustic metamaterials. Langmuir 2015, 31, 3215-3221.

${ }^{10} \mathrm{Li}$, J.; Chan, C.T.. Double-negative acoustic metamaterial. Phys. Rev. E, 2004, 70, 055602(R)

${ }^{11}$ Brunet, T.; Leng, J.; Mondain-Monval, O. Soft acoustic metamaterials. Science 2013, 342, 323-324.

${ }^{12}$ Brunet, T.; Merlin, A.; Mascaro, B.; Zimny, K.; Leng, J.; Poncelet, O.; Aristégui, C.; Mondain-Monval, O. Soft 3D acoustic metamaterials with negative index. Nat. Mater. 2015, 14, 384-388.

13 Raffy, S.; Mascaro, B.; Brunet, T.; Mondain-Monval, O.; Leng, J. A soft 3D metafluid with dual-band negative refractive index. Adv. Mater. in press.

${ }^{14}$ Cox, J. E. Silicone foam and method for making it. U.S. Patent 4859712 A, August 22, 1989.

15 Tebboth, M.; Jiang, Q.; Kogelbauer, A.; Bismarck, A. Inflatable Elastomeric Macroporous Polymers Synthesized from Medium Internal Phase Emulsion Templates. ACS Applied Materials \& Interfaces, 2015, 7, 19243-19250.

${ }^{16}$ Grosse, M.-T.; Lamotte, M.; Birot, M; Deleuze, H. Preparation of microcellular polysiloxane monoliths. $J$. Polym. Sci. A Polym. Chem., 2008, 46, 21-32.

17 Silverstein, M. S. PolyHIPEs: Recent advances in emulsion-templated porous polymers. Prog. Polym. Sci. 2014, 39, 199- 234.

${ }^{18}$ Pulko, I.; Krajnc, P. High internal phase emulsion templating - a path to hierarchically porous functional polymers. Macromol. Rapid Commun. 2012, 33, 1731-1746.

19 Barby, D.; Haq, Z. Low density porous cross-linked polymeric materials and their preparation and use as carriers for included liquids. U.S. Patent 4522953 A, June 11, 1985.

${ }^{20}$ Williams, J. M.; Wrobelski, D. A. Spatial distribution of the phases in water-in-oil emulsions. Open and closed microcellular foams from cross-linked polystyrene. Langmuir, 1988, 4, 656-662.

${ }^{21}$ S. Manley, N. Graeber, Z. Grof, A. Menner, G. F. Hewitt, F. Stepanek and A. Bismarck, Soft Matter, 2009, 5, 4780-4787.

${ }^{22}$ Cameron, N. R.; Sherrington, D. C. Adv. Polym. Sci. 1996, 126, 163-214.

${ }^{23}$ Menner, A.; Bismarck, A. New evidence for the mechanism of the pore formation in polymerising high internal phase emulsions or why polyHIPEs have an interconnected pore network structure. Macromol. Symp. 2006, 242, 19-24.

${ }^{24}$ Fischer-Cripps, A. C. Introduction to contact mechanics. Springer Science \& Business Media. New York 2007.

${ }^{25}$ Still, T.; Oudich, M.; Auerhammer, G. K.; Vlassopoulos, D.; Djafari-Rouhani, B.; Fytas, G.; Sheng, P. Soft silicone rubber in phononic structures: Correct elastic moduli. Phys. Rev. B 2013, 88, 094102.

${ }^{26} \mathrm{n}_{\mathrm{D}}(\mathrm{PDMS})=1.42, \mathrm{n}_{\mathrm{D}}($ water $)=1.33, \mathrm{n}_{\mathrm{D}}($ air $)=1.00$.

${ }^{27}$ Wheeler, T. D.; Stroock, A. D. The transpiration of water at negative pressures in a synthetic tree. Nature, 2008, 455, 208-212.

${ }^{28}$ Mark, J. E. Some interesting things about polysiloxanes. Acc. Chem. Res. 2004, 37, 946-953.

${ }^{29}$ Vincent, O.; Marmottant, P.; Quinto-Su, P. A.; Ohl, C. D. Birth and growth of cavitation bubbles within water under tension confined in a simple synthetic tree. Phys. Rev. Lett., 2012, 108, 184502. 
${ }^{30}$ Mastrangelo, C.H.; Hsu, C.H. Mechanical stability and adhesion of microstructures under capillary forces. I. Basic theory, and Mechanical stability and adhesion of microstructures under capillary forces. II. Experiments. Microelectromech. Syst. 1993, 2, 33 - 55.

${ }^{31}$ Decker, C. Photoinitiated cross-linking polymerisation. Prog. Polym. Sci. 1996, 21, 593-650. 


\section{TOC:}

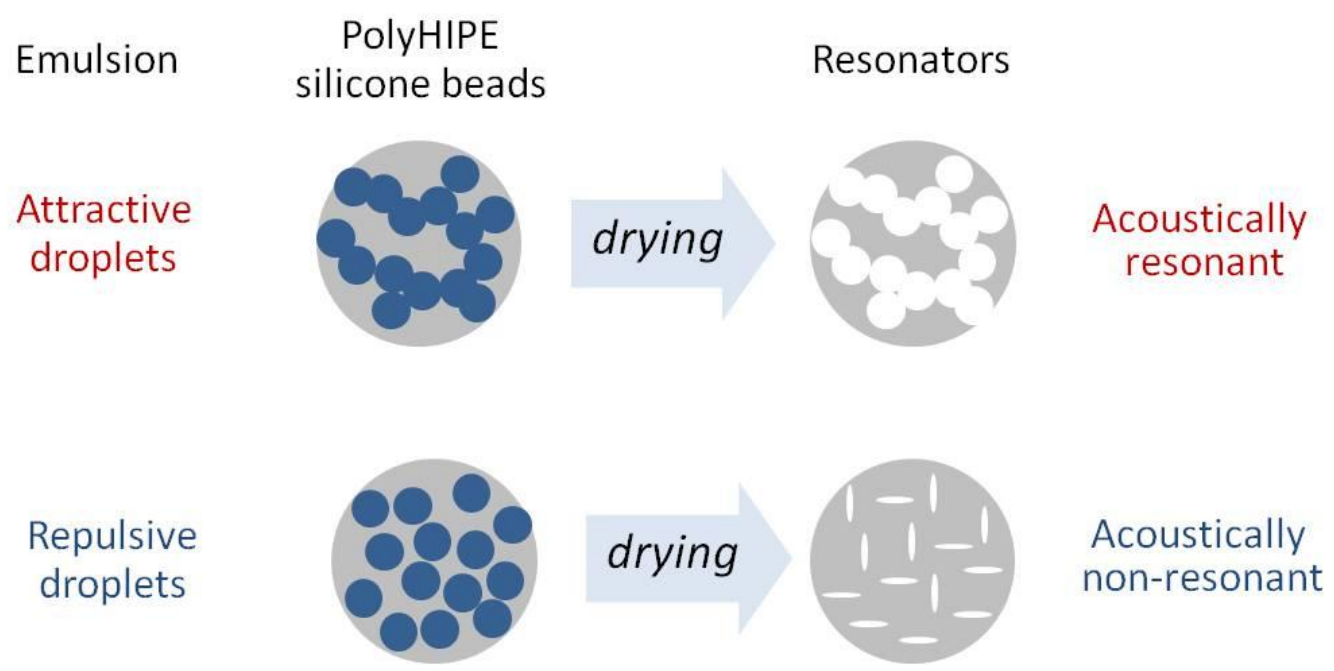

